Revista Brasileira de Agricultura Irrigada v.10, nº.5, p. 925 - 934, 2016

ISSN 1982-7679 (On-line)

Fortaleza, CE, INOVAGRI - http://www.inovagri.org.br

DOI: $10.7127 /$ rbai.v10n500469

Protocolo 469.16 - 11/08/2016 Aprovado em 29/09/2016

\title{
VELOCIDADE DE INFILTRAÇÃO DA ÁGUA NO SOLO CULTIVADO POR MILHO DOCE COM COBERTURA DE CROTALÁRIA
}

Igor Leonardo Nascimento Santos ${ }^{1}$, Raimundo Rodrigues Gomes Filho ${ }^{2}$, Clayton Moura de Carvalho $^{2}$, Ketylen Vieira Santos ${ }^{1}$, Débora Thalita Brito Oliveira ${ }^{1}$ e Leno Gonzaga Souza ${ }^{1}$

\section{RESUMO}

Este trabalho foi realizado em uma área experimental localizada na Estação Experimental da Universidade Federal de Sergipe - UFS, localizada aproximadamente a $20 \mathrm{Km}$ de Aracaju. Objetivou-se comparar a velocidade de infiltração de um Argissolo vermelho amarelo em diferentes manejos de uso do solo e com o cultivo de crotalária (Crotalariajuncea) como cobertura em sucessão à cultura do milho doce (Zeamays L.), analisando o impacto do uso agrícola a velocidade de infiltração de água no solo e a adequação dos modelos de Horton (1940), Kostiokov (1932), Kostiokov-Lewis (1945) e Phillip (1957). Os manejos de uso do solo consistiam de cultivo convencional, cultivo mínimo e plantio direto. A área foi dividida em três faixas, sendo que cada faixa contém um tipo de manejo, dividida em nove subparcelas que medem aproximadamente $60 \mathrm{~m}^{2}$, havendo três repetições distribuídas ao acaso. A velocidade de infiltração foi determinada com a metodologia dos duplos anéis concêntricos. Foi utilizado o software VIBK para a determinação dos valores obtidos e estimados da velocidade de infiltração da água no solo. Concluiu-se que o sistema de plantio direto proporcionou maiores taxas de infiltração da água no solo quando comparado com o convencional e mínimo. Os modelos de estimativa se ajustaram melhores em solos que sofreram menos ação antrópica e o modelo de Philips se adequou melhor as condições do local.

Palavras-chave: plantio direto, plantio convencional, cultivo mínimo, modelos de estimativa da infiltração.

\section{WATER INFILTRATION RATE IN SOIL GROWN IN SWEET CORN WITH CROTALARIA COVERAGE}

\footnotetext{
ABSTRACT

This work was carried out in an experimental area located in the Experimental Station of the Federal University of Sergipe - UFS, located about $20 \mathrm{~km}$ from Aracaju. This study aimed to

1 Graduando em Engenharia Agrícola, UFS, e-mails: igorsantos1993@gmail.com; kvsantos@gmail.com; deboratbo@gmail.com; lenogsouza@gmail.com

2 Doutor em Engenharia Agrícola, Professor do PRORH/UFS, e-mails: rrgomesfilho@hotmail.com; carvalho_cmc@yahoo.com.br
} 
compare the speed of infiltration of a red yellow Argisol in different managements of land use and the crotalaria cultivation (Crotalariajuncea) as succession coverage to sweet corn (Zeamays L.), analyzing the impact of agricultural use water infiltration rate of the soil and the adequacy of Horton models (1940), Kostiokov (1932), Kostiokov-Lewis (1945) and Phillip (1957). The management of land use consisted of conventional tillage, minimum tillage and no tillage. The area was divided into three tracks, each track contains a type of management, divided into nine sub-plots measuring approximately $60 \mathrm{~m}^{2}$, with three replicates randomly distributed. The rate of infiltration is determined by the methodology of the dual concentric rings. VIBK software was used for the determination of estimated values obtained and the rate of water infiltration into the soil. It was concluded that the tillage system provided higher water infiltration rates in soil compared to the conventional and minimum. The estimation models set best in soils that suffered less human action and the model Philips is best suited local conditions.

Keywords: tillage, conventional tillage, minimum cultivation, infiltration estimation models.

\section{INTRODUÇÃO}

Para que haja uma gestão segura dos recursos naturais, a análise quantitativa e a interpretação dos atributos físicos e físicohídricos e das principais alterações geradas na qualidade do solo são fundamentais (STEFANOSKI et al., 2013).

Segundo Gondim et al. (2010), o conhecimento da taxa de infiltração da água no solo é de fundamental importância para definir técnicas de conservação do solo, planejar e delinear sistemas de irrigação e drenagem, bem como auxiliar na composição de uma imagem mais real da retenção da água e aeração no solo. A infiltração é o processo pelo qual a água atravessa a superfície do solo (BRANDÃO et al., 2006b).

Segundo Bernardo et al. (2008), a velocidade de infiltração da água no solo (VI) é a velocidade com que a água se infiltra no solo através de sua superfície, sendo expressa por unidade indicativa de altura de lâmina d'água ou volume de água infiltrada em determinado perfil do solo por unidade de tempo como $\mathrm{mm} \mathrm{h}^{-1}, \mathrm{~cm}$ $\mathrm{h}^{-1}$, ou L s ${ }^{-1}$. Acrescenta Gondim et al. (2010) que a taxa de entrada de água no solo decresce com o tempo em função do umedecimento do perfil, assumindo um valor mínimo quase constante denominado de velocidade de infiltração básica (VIB).

A velocidade de infiltração é muito influenciada pelas condições da superfície, do perfil e conteúdo inicial de água do solo e os processos de erosão hídrica são fortemente afetados pelos materiais de superfície, topografia, sazonalidade das chuvas e cobertura vegetal e podem ser potencializados por alterações na textura, estrutura, porosidade, conteúdo de matéria orgânica, devido ao uso das terras e seus respectivos manejos e presença e/ou construção de estradas (PANACHUKI et al., 2006; PEREIRA; TEIXEIRA FILHO, 2009; FEITOSA FILHO, 2009; GONDIM et al., 2010).

A taxa de infiltração de água no solo é uma das propriedades que melhor reflete as condições físicas do solo, sua qualidade e estabilidade estrutural. Sendo assim, formas de manejos mais agressivas, como as que vêm sendo utilizadas convencionalmente. Aração e gradagem compactam o solo e refletem diretamente na VIB, deixando-a mais lenta, em um solo tratado com um manejo mais brando, como o plantio direto, a compactação é menos agressiva, o que faz com que a VIB desse solo seja maior.

A cobertura vegetal é outra variável que pode influenciar a infiltração da água, ter sempre uma cobertura quando o solo não esta sendo usado para fins agrícolas é importante, pois ela protege o solo contra o impacto direto das gotas da chuva, que podem favorecer o selamento da superfície do mesmo, também protege contra a radiação direta dos raios solares, mantendo a umidade do solo por mais tempo, ela também aumenta a microporosidade, que vai influenciar diretamente nos caminhos preferenciais da água durante a infiltração. 
A infiltração também tem uma grande importância na irrigação e drenagem, pois ela auxilia durante um projeto, limitando a intensidade de aplicação de água na irrigação (ARAÚJO FILHO; RIBEIRO, 1996; POTT, 2001). Mas por ter um teste de duração muito longa, muitas vezes a VIB não é obtida, ou em outros casos, quando é necessária a descrição do fenômeno através de curvas e equações, o resultado é de baixa qualidade, que pode ser causado pela utilização de um método não compatível ou realizado de forma erronia.

O presente trabalho teve como objetivo comparar a velocidade de infiltração de um Argissolo vermelho amarelo em diferentes manejos de uso do solo e cultivo de crotalária (Crotalariajuncea) como cobertura em sucessão à cultura do milho doce (Zeamays L.), analisando o impacto do uso agrícola e a adequação dos modelos de Horton (1940), Kostiokov (1932), Kostiokov-Lewis (1945) e Phillip (1957).

\section{MATERIAL E MÉTODOS}

\section{Descrição da área}

O estudo foi realizado em uma área experimental localizada na Estação Experimental da Universidade Federal de Sergipe - UFS, localizada aproximadamente a $20 \mathrm{Km}$ de Aracaju, cujas coordenadas geográficas de Greenwich são $10^{\circ} 19^{\prime} \mathrm{S}$ de latitude, $36^{\circ} 39^{\prime} \mathrm{O}$ de longitude, em solo classificado como Argissolo Vermelho Amarelo. A região possui clima, de acordo com a classificação de Köppen, do tipo As'Tropical chuvoso com verão seco e pluviometria em torno de $1200 \mathrm{~mm}$ anuais, com chuvas concentradas nos meses de abril a setembro.

A área experimental tem aproximadamente $3000 \mathrm{~m}^{2}$ e foi instalada no ano de 2001, onde vem sendo estudado o comportamento dos sistemas de cultivo: cultivo convencional (composto de gradagem com grade niveladora de discos + aração com arado de discos + gradagem), cultivo mínimo (composto de 1 ou 2 gradagens com grade niveladora de discos, sendo que a segunda gradagem somente realizada quando há incidência considerável de plantas daninhas) e plantio direto (consistindo do não revolvimento do solo) e cultivo crotalária (Crotalariajuncea) (Figura 1) em sucessão à cultura do milho doce (Zeamays L.) variedade Biomatrix BM 3061.

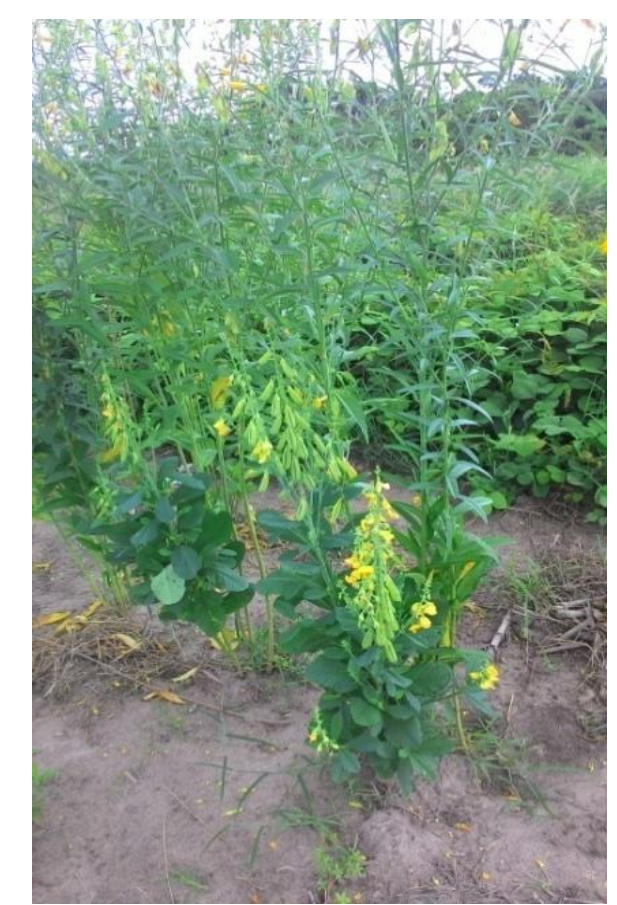

Figura 1. Parcela de plantio

convencional com cobertura de Crotalária.

A área foi dividida em três faixas, utilizando-se o esquema de faixas experimentais, sendo que cada faixa contém um tipo de manejo, cada faixa foi dividida em três subparcelas que medem aproximadamente 60 $\mathrm{m}^{2}$.

\section{Determinação da taxa de infiltração}

As coletas de dados da velocidade de infiltração foram realizadas quando o solo encontrava-se com pouca umidade, caso houvesse chuva, esperava-se um intervalo de pelo menos dois dias, para que a umidade baixasse, assim, as curvas que seriam geradas pelos dados teriam características mais acentuadas, tornando a aplicação dos modelos matemáticos mais precisos. Foram utilizados infiltrômetros de anéis concêntricos (IANC) para realização do teste como pode ser observado na Figura 2. 


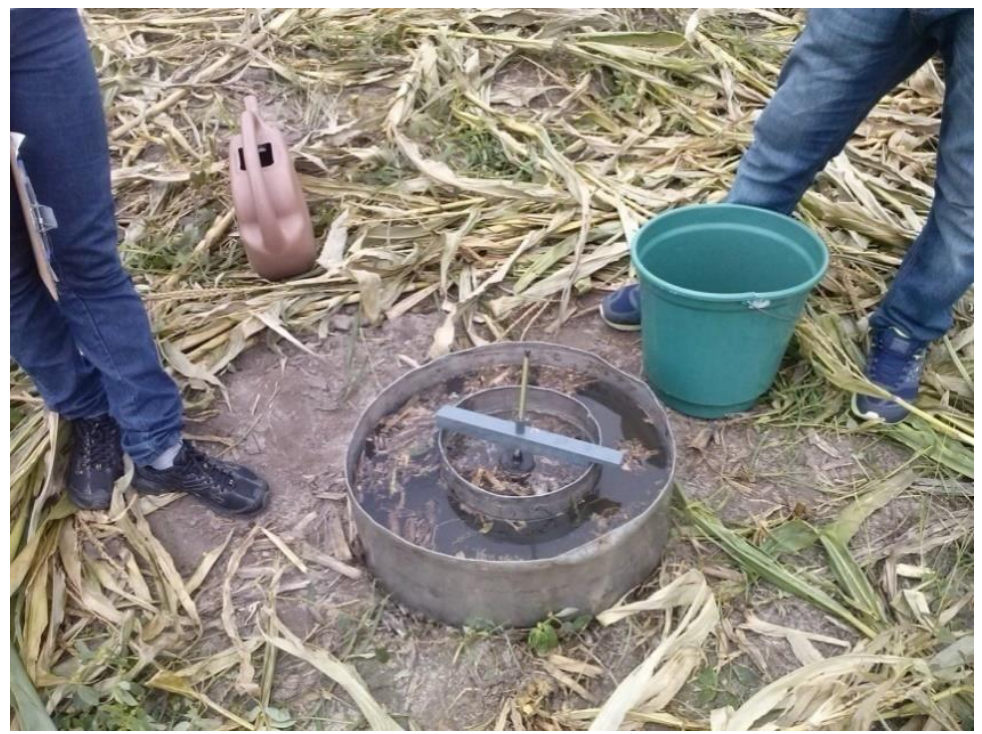

Figura 2. Realização de um teste de infiltração na área de plantio direto.

Esse instrumento consiste em dois
anéis concêntricos, sendo o maior com diâmetro de 40 centímetros e o menor com diâmetro de 20 centímetros como demonstrado na Figura 3, ambos com altura de 40 centímetros.

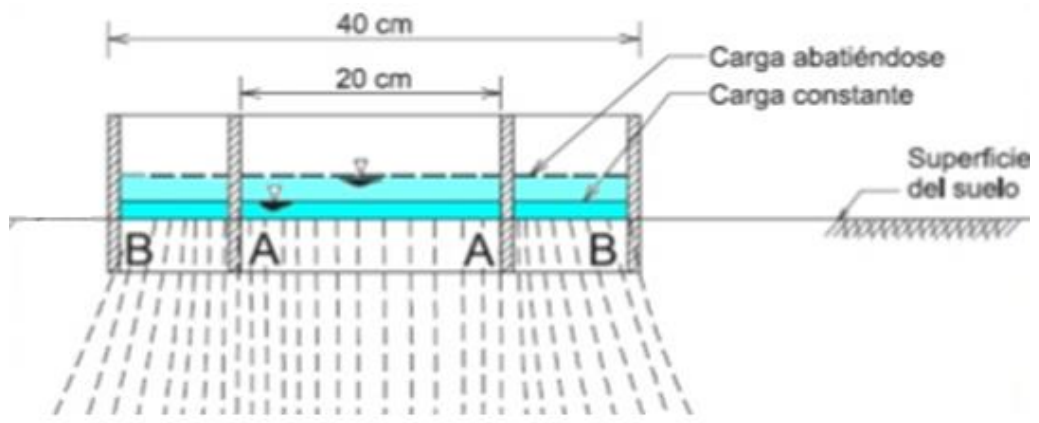

Figura 3. Diagrama do funcionamento dos anéisinfiltrômetros.

A determinação da infiltração se processa pela medida da altura de água infiltrada no cilindro menor (interno) em tempos sucessivos de leituras. O cilindro externo tem a função de eliminar a infiltração lateral do cilindro interno. Ambos os anéis foram instalados aproximadamente cinco centímetros de profundidade. A lâmina de água foi mantida a uma altura que variava entre 10 e $15 \mathrm{~cm}$ e era constantemente reabastecida ao fim de cada contagem de tempo previamente estabelecida, repetindo os intervalos de 20 minutos até que fosse alcançado o critério estabelecido para o fim do teste, ou seja, a lâmina de água infiltrada no cilindro interno se tornasse constante, com o intervalo de tempo e se repetisse pelo menos três vezes. Os testes duraram entre 122 e 182 minutos.

\section{Modelos para descrição do fenômeno}

Para tornar o processo de cálculo $\mathrm{e}$ plotagem mais rápido e eficaz, foi utilizado o software VIBK (SANTOS et al., 2015).O algoritmo do VIBK foi desenvolvido em uma Graphical User Interface (GUI) do software Matlab, que por ter uma estrutura de dados vetoriais, recursos de programação e ferramentas GUI, se torna uma ferramenta poderosa para solução de problemas em diversos campos(HANSELMAN; LITTLEFIELD, 2003).

Para os modelos de Horton, Kostiakov, Kostiakov-Lews e Philips foram utilizadas as equações de 1 a 8 , descritas por Brandão (2006a).

\section{Modelo de Horton:}




\section{COBERTURA DE CROTALÁRIA}

$$
\begin{aligned}
& \mathrm{I}=\mathrm{i}_{\mathrm{f}} \mathrm{t}+\frac{(\mathrm{ii}-\mathrm{if})}{\beta}\left(1-e^{-\beta \mathrm{t}}\right) \\
& \mathrm{VI}=\frac{d I}{d t}=\mathrm{i}_{\mathrm{f}}+\left(\mathrm{i}_{\mathrm{i}}-\mathrm{i}_{\mathrm{f}}\right) e^{-\beta \mathrm{t}}
\end{aligned}
$$

Modelo de Kostiakov:

$$
\begin{aligned}
& \mathrm{I}=k \mathrm{t}^{\alpha} \\
& \mathrm{VI}=\frac{d I}{d t}=\mathrm{k} \alpha \mathrm{t}^{\alpha-1}
\end{aligned}
$$

Modelo de Kostiakov-Lewis:

$$
\begin{aligned}
& \mathrm{I}=k \mathrm{t}^{\alpha}+\mathrm{i}_{\mathrm{f}} \\
& \mathrm{VI}=\frac{d I}{d t}=\mathrm{k} \alpha \mathrm{t}^{\alpha-1}+\mathrm{i}_{\mathrm{f}}
\end{aligned}
$$

Modelo de Philip:

$$
\begin{aligned}
& \mathrm{I}=\mathrm{f}_{1} \mathrm{t}^{1 / 2}+\mathrm{f}_{2} \mathrm{t} \\
& \mathrm{VI}=\frac{d I}{d t}=\frac{1}{2} \mathrm{f}_{1} \mathrm{t}^{-1 / 2}+\mathrm{f}_{2}
\end{aligned}
$$

Onde, I é a velocidade de infiltração $\left(\mathrm{mmh}^{-1}\right)$, ii velocidade de infiltração inicial, if velocidade de infiltração básica(VIB), t representa o tempo acumulado e $k, \alpha, \beta, \mathrm{f}_{1}, \mathrm{f}_{2}$ são constantes obtidas, empiricamente, através de regressões.

Para avaliar o desempenho entre os valores da infiltração obtidos em campo em relação os valores calculados através dos modelos de Kostiakov, Horton e KostiakovLewis e Philip, análises comparativas dos resultados foram realizadas estatisticamente através do coeficiente determinação $\left(\mathrm{R}^{2}\right)$. Para tornar a análise mais eficiente, também foram calculados os índices estatísticos das equações $9,10,11$, respectivamente, coeficiente de massa residual (CMR), coeficiente de ajuste (CA) e eficiência (EF) conforme descrito por Brito et al. (2009).

$$
\begin{gathered}
\mathrm{CMR}=\frac{\sum_{i=1}^{n} O_{i}-\sum_{i=1}^{n} P_{i}}{\left(\sum_{i=1}^{n} O_{i}\right)} \\
\mathrm{CA}=\frac{\sum_{i=1}^{n}\left(O_{i}-\bar{O}\right)^{2}}{\sum_{i=1}^{n}\left(P_{i}-\bar{O}\right)^{2}} \\
\mathrm{EF}=\frac{\sum_{i=1}^{n}\left(O_{i}-\bar{O}\right)^{2}-\sum_{i=1}^{n}\left(P_{i}-O_{i}\right)^{2}}{\sum_{i=1}^{n}\left(O_{i}-\bar{O}\right)^{2}}
\end{gathered}
$$

em que:

$\boldsymbol{O} \boldsymbol{i}$ - representa os valores observados,

$\boldsymbol{P i}$ - os estimados, n é o número de observações,

$\overline{\boldsymbol{O}}$ - a média aritmética das observações,

$\overline{\boldsymbol{P}}$ - a média aritmética dos valores estimados.

\section{RESULTADOS E DISCUSSÃO}

As VIBs obtidas em campo foram 123 e 87 $\mathrm{mm} \mathrm{h}^{-1}$ para plantio direto e mínimo respectivamente, consideradas velocidades muito altas pela classificação de Bernardo et al. (2008). Já na área de plantio convencional a VIB encontrada foi $18 \mathrm{~mm} \mathrm{~h}^{-1}$, que pela classificação ainda é uma alta velocidade (Tabela 1).

Tabela 1. Classificação do solo a partir de sua VIB.

\begin{tabular}{cc}
\hline Tipos de solo & VIB $\left(\mathbf{m m ~ h}^{\mathbf{- 1}}\right)$ \\
\hline Solo de VIB muito alta & $>30$ \\
Solo de VIB alta & $15-30$ \\
Solo de VIB média & $5-15$ \\
Solo de VIB baixa & $<5$ \\
\hline
\end{tabular}

Fonte: Bernardo et al. (2008)

Assim como foi evidenciado pelos autores Gonçalves e Moraes (2012) e Sobrinho et al. (2003), a maior VIB foi encontrada na área onde o manejo era plantio direto, pois com esse tratamento o solo normalmente é menos compactado, assim a existência de macroporos é favorecida, facilitando a infiltração, pois como já foi relatado por Sobrinho et al. (2000), o uso excessivo de grades modifica o volume de macroporos, formando o denominado "pé de 
grade", que é uma camada adensada entre 10 e $30 \mathrm{~cm}$ de profundidade. Da mesma maneira, o pior resultado foi encontrado na área de plantio convencional, que pode ter sido causada pela compactação do solo.
Agora analisando os dados da Tabela 2, que são os resultados estatísticos das taxas de infiltração obtidas e calculadas para todos os manejos.

Tabela 2.VIBs e índices estatísticos para os três tipos de manejo

\begin{tabular}{|c|c|c|c|c|c|}
\hline Modelo & $\operatorname{VIB}\left(\mathrm{mm}^{2} \mathrm{~h}^{-1}\right)$ & $\mathrm{R}^{2}$ & CMR & $\mathrm{CA}$ & $\mathrm{EF}$ \\
\hline & \multicolumn{5}{|c|}{ Plantio direto } \\
\hline Observados & 123 & 1 & 0 & 1 & 1 \\
\hline Horton & 123,01 & 0,9665 & 0,0136 & 1,1279 & 0,9328 \\
\hline Kostiakov & 111,19 & 0,9761 & 0,0100 & 1,2601 & 0,9450 \\
\hline Kostiakov-Lewis & 135,17 & 0,9797 & 0,1163 & 3,7001 & 0,6315 \\
\hline \multirow[t]{2}{*}{ Philip } & 118,32 & 0,9824 & 0,0000 & 1,0361 & 0,9824 \\
\hline & \multicolumn{5}{|c|}{ Cultivo mínimo } \\
\hline Observados & 87 & 1 & 0 & 1 & 1 \\
\hline Horton & 87 & 0,9563 & 0,0144 & 1,1738 & 0,9129 \\
\hline Kostiakov & 75,23 & 0,9603 & 0,0285 & 1,7593 & 0,8775 \\
\hline Kostiakov-Lewis & 94,27 & 0,9951 & 0,1760 & 4,9806 & 0,5332 \\
\hline \multirow[t]{2}{*}{ Philip } & 70,49 & 0,9823 & 0,0000 & 1,0364 & 0,9649 \\
\hline & \multicolumn{5}{|c|}{ Cultivo convencional } \\
\hline Observados & 18 & 1 & 0 & 1 & 1 \\
\hline Horton & 18 & 0,9899 & 0,1562 & 2,1509 & 0,7335 \\
\hline Kostiakov & 12,82 & 0,9581 & 0,1189 & 2,6761 & 0,4418 \\
\hline Kostiakov-Lewis & 19,02 & 0,9879 & 0,3888 & 7,1234 & 0,0696 \\
\hline Philip & 3,649 & 0,9538 & 0,0000 & 1,0992 & 0,7799 \\
\hline
\end{tabular}

Em relação aos modelos, o que melhor ajustou a VIB em todos os tipos de manejos foi o de Horton, pois a VIB calculada foi igual à obtida no campo, o que é facilmente explicado pela forma que a equação evolui, o primeiro termo é a VIB real obtida em campo, já o segundo termo é responsável por representar o fenômeno até chegar à VIB, de acordo com que o tempo aumenta o segundo termo decresce, para um tempo muito grande esse termo tende a zero, assim a VIB se aproxima muito da obtida em campo. Porém, o segundo termo da equação de método também causa uma dificuldade no ajuste do início da curva, já que a curva de uma exponencial tem um desenho bem característico, o que faz alguns pontos ficarem fora da curva caso o decaimento da taxa de infiltração seja muito acentuada. PAIXÃO et al., 2009; SCHREINER et al., 2010; TOMASINI, et al., 2010; SANTOS, 2014, também evidenciaram em seus estudos que a equação de Horton é perfeitamente aderente sendo a que melhor expressa a velocidade de infiltração de água no solo descrito através de modelos matemáticos.

Em relação ao coeficiente de determinação $\left(\mathrm{R}^{2}\right)$, em todos os modelos de todos os manejos, os resultados foram satisfatórios, todos obtiveram mais de $95 \%$ nesse teste, no manejo plantio direto o método de Philips alcançou o melhor resultado, no plantio mínimo foi o modelo de KostiakovLewis e no convencional os melhores resultados foram dos métodos Kostiakov-Lewis e Horton.

Para o método de Philips houve um resultado bastante interessante no coeficiente de massa residual (CMR), pois em todos os manejos esse coeficiente se aproximou bastante do ideal, ou seja, a curva foi se ajustando muito bem, de maneira que praticamente não houve resíduos, também se pode afirmar que para esse método o volume de água infiltrada é a mesma que a obtida em campo. Os outros modelos não 
tiveram um resultado tão satisfatórios de CMR como o Phillips, porém é possível verificar que quanto mais agressivo é o uso do solo, há uma dificuldade maior no ajuste da curva pelos métodos, gerando resultados mais conturbados e CMR mais distantes de 1 .

Sobre o coeficiente de ajuste (CA), os resultados foram satisfatoriamente semelhantes em todos os métodos, com exceção o de Kostiakov-Lewis, que não conseguiu se adequar ao solo. E mais uma vez o método de Philip obteve melhores resultados em todos os manejos.

Por último, foi estudada a eficiência dos modelos (EF). Nesse quesito todos os testes, com exceção do de Kostiakov-Lewis, obtiveram bons resultados no manejo de plantio direto e cultivo mínimo. Já no manejo convencional, os resultados não foram satisfatórios e nenhum dos métodos conseguiu representar o fenômeno com uma eficiência relevante, ratificando que o manejo convencional pode trazer sérios
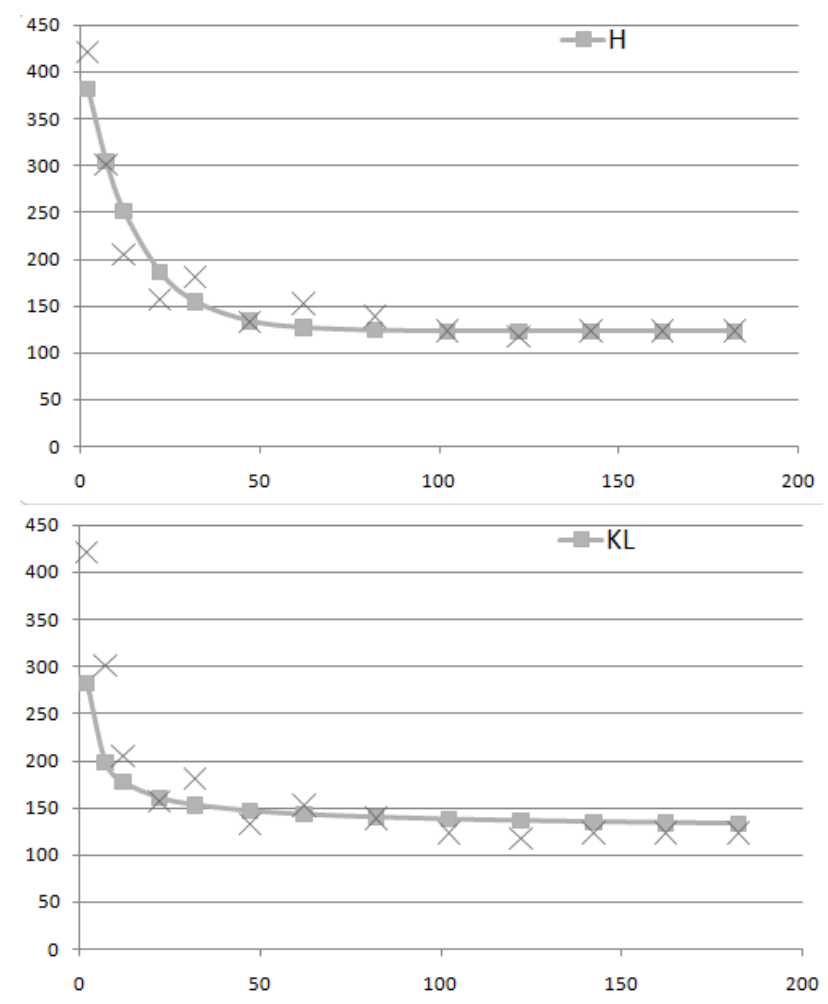

Figura 4. Curvas e taxas de infiltração observadas e estimadas na área do plantio direto. Onde a ordenada horizontal representa o tempo em horas e a vertical a infiltração em $\mathrm{mm} \mathrm{h}^{-1}$ e H-Horton, K-Kostiakov, KLKostiakov-Lewis e P-Philips. prejuízos à estabilidade de um solo. Outra observação foi a baixa eficiência em todos os parâmetros estatísticos do modelo de KostiakovLewis para todos os manejos, sendo satisfatório apenas quanto ao coeficiente de determinação $\left(\mathrm{R}^{2}\right)$, deixando evidente que apenas a análise do coeficiente de determinação não torna esse tipo de estudo estatístico confiável, além de mostrar que esse modelo tende a subestimar o valor a partir de 10 minutos de experimento, fato já evidenciado por Sobrinho et al. (2003), como também superestimar o valor da VIB.

Também se pode observar que existe um pé-de-grade no manejo convencional através da curva, pois ao se comparar as curvas geradas no plantio direto (Figura 4) e as do cultivo convencional (Figura 5) pode-se observar uma mudança brusca no coeficiente da curva convencional em aproximadamente os cinco minutos de teste, fato não evidenciado nas curvas do plantio direto.
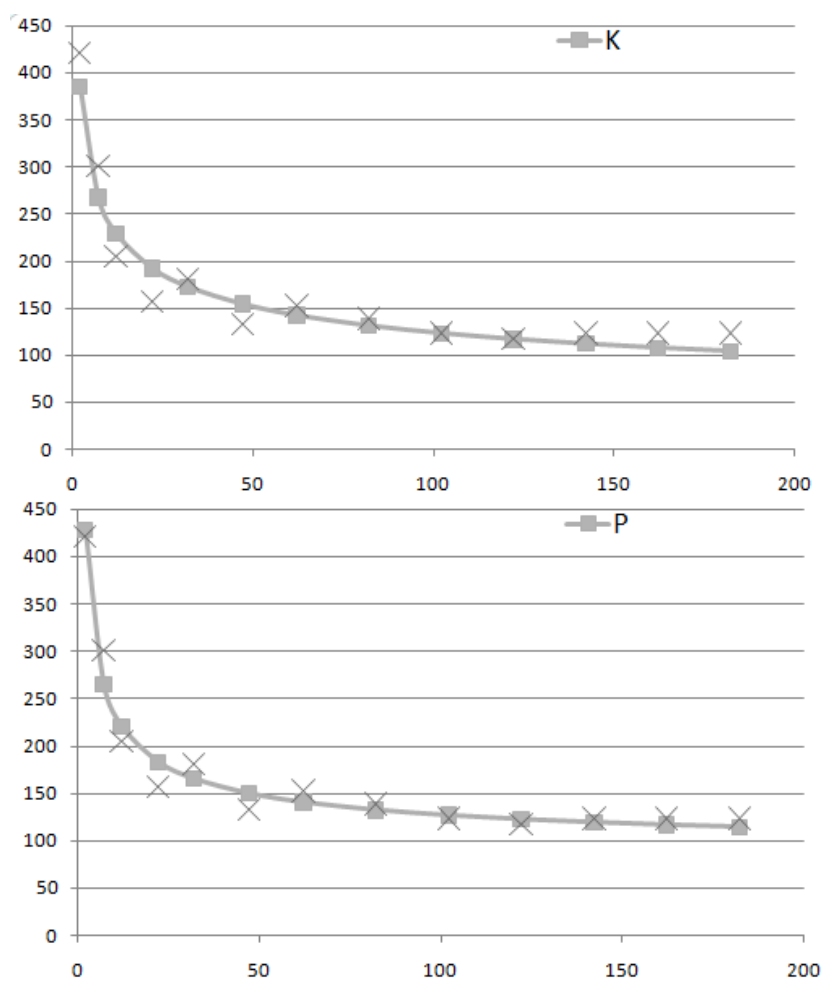

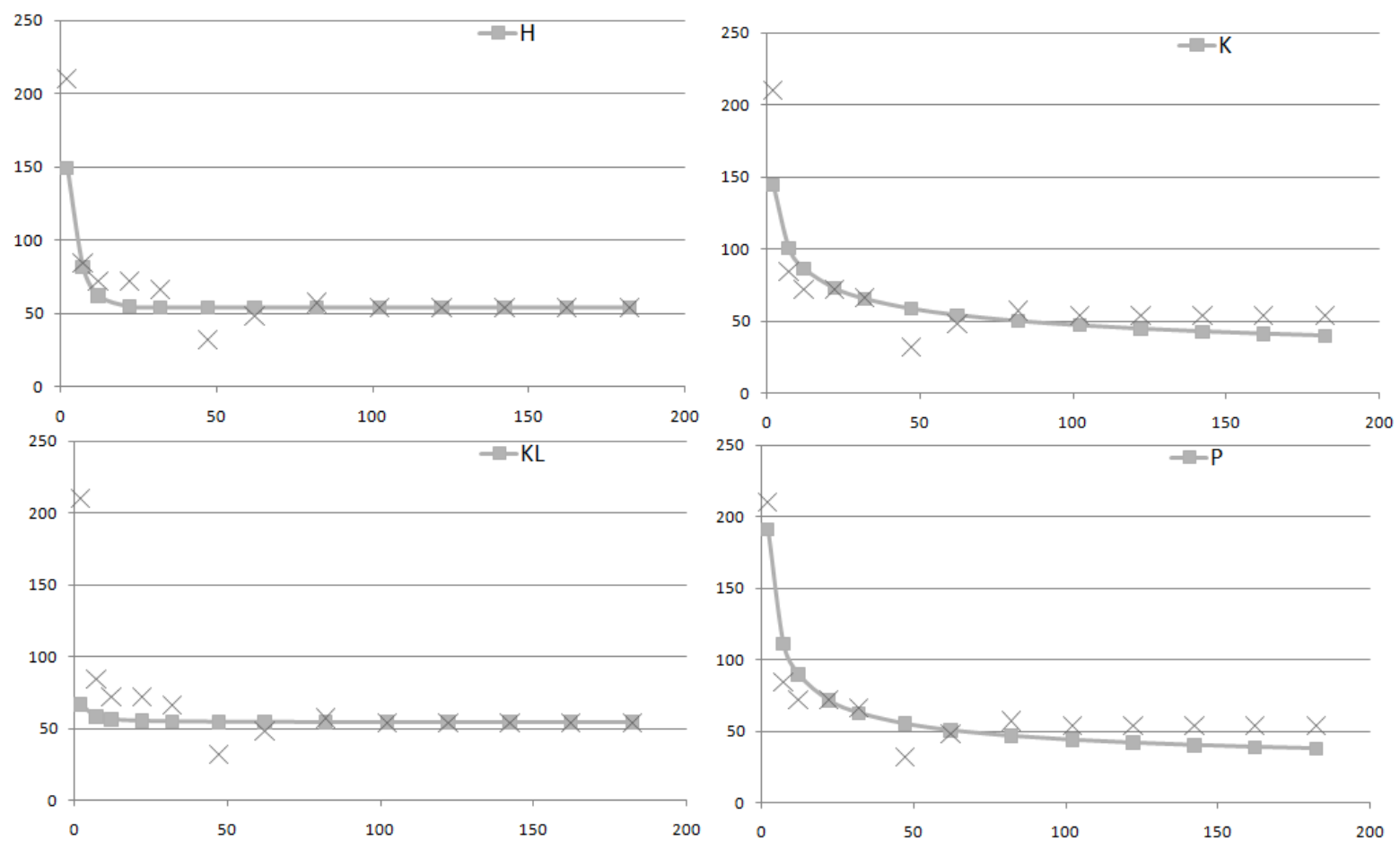

Figura 5. Curvas e taxas de infiltração observadas e estimadas na área de cultivo convencional. Onde a ordenada horizontal representa o tempo em horas e a vertical a infiltração em $\mathrm{mm} \mathrm{h}^{-1}$ e H-Horton, K-Kostiakov, KLKostiakov-Lewis e P-Philips.

Analisando o comportamento do desenho das curvas é possível notar fatos já relatados por Sobrinho et al.(2003) e Tomasini et al.(2010), existe uma tendência dos modelos em superestimar a velocidade de infiltração no inicio do teste e em contra partida, os modelos tende a subestimar a velocidade no final do teste, com exceção do Kostiakov-Lewis que continua a superestimar.

Além disso, é fácil perceber que os dados obtidos na área de plantio direto são mais estáveis que a do plantio convencional, seguindo um desenho mais suave.

\section{CONCLUSÃO}

1. Dentro das limitações de cada modelo, todos geraram resultados estatisticamente equivalentes, com exceção ao modelo de Kostiakov-Lewis.

2. O modelo matemático que, em média, obteve melhores resultados foi o de Philip.

3. Um solo manejado sob plantio direto, em relação aos outros manejos, mostrou-se mais favorável quanto à infiltração de água no solo.

\section{REFERÊNCIAS}

ARAÚJO FILHO, J.C. de; RIBEIRO, M.R. Infiltração de água em Cambissolos do Baixio de Irecê (BA). Revista Brasileira de Ciência do Solo, v.20, n.3, p.363-370, 1996.

BERNARDO, S.; SOARES, A. A.; MANTOVANI, E. C. Manual de irrigação. 8. ed. Atual. e Ampl. Viçosa: UFV, 2008. 625 p.

BRANDÃO, V. S.; CECILIO, R. A.; PRUSKI, F. F.; SILVA, D. D. da. Infiltração da água no solo. 3. ed., atual. e ampl. Viçosa, MG: UFV, 2006a. 120 p.

BRANDÃO, V. S.; SILVA, D. D.; RUIZ, H. A.; PRUSKI, F. F.; SCHAEFER, C. E. G. R.; MARTINEZ, M. A.; MENEZES, S. J. M. C. Resistência hidráulica da crosta formada em solos submetidos a chuvas simuladas. Revista Brasileira de Ciência do Solo, v.30, p. 13-22, 2006b.

BRITO, A. dos S.; LIBARDI, P. L.; MOTA, J. C. A.; MORAES, S. O. Desempenho do 
tensiômetro com diferentes sistemas de leitura. Revista Brasileira de ciência do solo, v.33, n.1, p. 17-24, 2009.

FEITOSA FILHO, J. C. Infilttração acumulada d'água no solo (I) e velocidade de infiltração d'água no solo (VI). Areia: UFPB/CCA, 2009, 5p. (Apostila, Disciplina Irrigação e Drenagem/Relação SoloAgua_PlantaAtmosfera).

GONÇALVES, F. C.; MORAES, M. H. Porosidade e infiltração de água do solo sob diferentes sistemas de manejo. Irriga, v.17, n.3, p. 337, 2012.

GONDIM, T. M. de S.; WANDERLEY, J. A. C.; SOUZA, J. M. de; FEITOSA FILHO, J. C.; SOUSA, J. da S. Infiltração e velocidade de infiltração de água pelo método do infiltrômetro de anel em solo arenoargiloso. Revista Brasileira de Gestão Ambiental, v.4, n.1, p. 64-73, 2010.

HANSELMAN, D.; LITTLEFIELD, B. MATLAB 6: curso completo. São Paulo: Pearson, 2003 xvi, 676 p.

PAIXÃO, F. J. R.; ANTONIO R. S. ANDRADE, A. R. S.; AZEVEDO, C. A. V.; COSTA. T. L.; GUERRA, O. C. Ajuste da curva de infiltração por meio de diferentes modelos empíricos.Pesquisa Aplicada \& Agrotecnologia, v. 2, n. 1. 2009.

PANACHUKI, E.; SOBRINHO, T. A.; VITORINO, A. C. T.; CARVALHO, D. F.; URCHEI, M. A. Avaliação da infiltração de água no solo, em sistema de integração agricultura-pecuária, com uso de infiltrômetro de aspersão portátil. Acta Scientiarum Agronomy, v.28, n.1, p. 129- 137, 2006.

PEREIRA, V. R.; TEIXEIRA FILHO, T. Identificação das áreas susceptíveis aos processos erosivos em duas bacias do Sistema Cantareira por meio de diferentes cenários. Acta Scientiarum Agronomy, v. 31, n. 1, p. 155163, 2009.
POTT, C. A. Determinação da velocidade de infiltração básica de água no solo por meio de infiltrômetros de aspersão, de pressão e de tensão, em três solos do estado de São Paulo. Campinas, 2001, 77p. Dissertação (Mestrado), Instituto Agronômico de Campinas - IAC.

SANTOS, H. M. T. Estimativa da velocidade de infiltração básica (VIB) nos solos da sub-bacia do Rio Siriri-SE utilizando o modelo de Horton, Kostiakov-Lewis e Philip. 2014. 84 p. Dissertação (mestrado em Recursos Hídricos) Universidade Federal de Sergipe.

SANTOS, I. L. N.; GOMES FILHO, R. R.; SANTOS, K. V.; MASSARANDUBA, W.de M.; CARVALHO, C. M. de. Software vibk for estimated infiltration rate and water hydraulic conductivity in soil. In: Inovagri International Meeting, 3, 2015, Fortaleza. Anais... Fortaleza, INOVAGRI, 2015.

SCHREINER, D. T.; VOGELMANN, E. S.; PREVEDELLO, J.; REICHERT, J. M.; REINERT, D. J.; CONSENSA, C. O. B. Estimativa da infiltração de água no solo através de pedofunções em área de floresta plantada. Anais do VII Reunião Sul Brasileiro de ciência do solo. p. 1-6, 2010.

SOBRINHO, T. A.; PEIXOTO, P. P. P.; SOUZA, L. C. F.; FEDATTO, E.; MAPELI, N. C. Avaliação de alguns atributos do sistema solo- água-planta em plantio direto e convencional: Parte I. In: Congresso Brasileiro de Engenharia Agrícola, 30, 2000, Fortaleza. Resumos... Fortaleza, SBEA, 2000. CD-Rom

SOBRINHO, T. A.; VITORINO, A. C. T.; SOUZA, L. C. F. de; GONÇALVES, M. C.; CARVALHO, D. F. de. Infiltração de água no solo em sistemas de plantio direto e convencional. Revista Brasileira de Engenharia Agrícola e Ambiental, v.7, n.2, p. 191-196, 2003.

STEFANOSKI, D. C.; SANTOS, G. G.; MARCHÃO, R. L.; PETTER, F. A.; PACHECO, L. P. Uso e manejo do solo e seus impactos sobre a qualidade física. Revista 
Brasileira de Engenharia Agrícola e Ambiental, v. 17, n. 12, p. 1301-1309, 2013.

TOMASINI, B. A.; VITORINO, A. C. T.; GARBIATE, M. V SOUZA, C. M. A.; 4,
TEODORICO A. T. S. Infiltração de água no solo em áreas cultivadas com cana-de- -açúcar sob diferentes sistemas de colheita e modelos de ajustes de equações de infiltração. Engenharia Agrícola, v. 30, n. 6, p.1060-1070. 2010. 